\title{
Purification et quelques caractéristiques moléculaires du « composant-3 » des protéose-peptones
}

\author{
par \\ D. PAQUET et C. ALAIS* \\ avec la collaboration technique de F. AUBERT
}

\section{Ré s u mé}

Les protéose-peptones étaient isolées de lait cru ou de lactosérum acide par trois méthodes, puis examinées par électrophorèse sur gel de polyacrylamide. Elles présentaient des profils densitométriques presque identiques. Seule, la bande correspondant au composant-3 était colorée par le réactif de Schiff. La chromatographie liquide d'interactions hydrophobes sur colonne de phenylsepharose CL 4B était une bonne méthode pour fractionner les protéose-peptones en leurs composants principaux. Une chromatographie supplémentaire sur gel de séphacryl S 200 permettait de purifier le composant-3. L'analyse des acides aminés montrait que ce composant contenait moins de proline mais plus de sérine et de glycine que les protéose-peptones d'origine. L'électrophorèse sur gel de polyacrylamide en présence de dodecyl sulfate de sodium donnait un poids moléculaire voisin de 22000 . La sérine était identifiée comme acide aminé $\mathrm{N}$-terminal par dansylation. Le taux de phosphore était voisin de $1,1 \%$.

Mots clés

Protéose-peptones - Composant-3 - Fractionnement - Purification - Composition.

* Laboratoire de Biochimie Appliquée, Faculté des Sciences, Université de Nancy I, B.P. 239 - 54506 Vandœuvre-les-Nancy. 


\title{
S u m m a r y
}

\author{
PURIFICATION AND SOME MOLECULAR CHARACTERISTICS \\ OF THE «COMPONENT-3» OF PROTEOSE-PEPTONES
}

The proteose-peptones were isolated from skimmilk or acid whey by mean of three methods, then analyzed by polyacrylamide gel electrophoresis. They showed almost identical densitometric diagrams. Only the band corresponding to component-3 gave characteristic colour with the Schiff reagent. The hydrophobic interaction chromatography on phenyl-sepharose CL $4 B$ column was a suitable method to separate the major proteose-peptone components. A further gel filtration on Sephacryl S 200 allowed us to purify the component-3. The amino acid analysis showed that the component-3 contained less proline but more serine and glycine than the original proteose-peptones. Polyacrylamide gel electrophoresis in the presence of sodium dodecyl sulphate gave a molecular weight value near 22000 . The N-terminal residue was found to be serine by dansylation. The phosphorus content was near $1,1 \%$.

Key words

Proteose-peptones - Fractionation - Component-3 - Purification - Composition.

\section{INTRODUCTION}

Les protéose-peptones du lait de vache forment la fraction protéique soluble après chauffage du lait à $95^{\circ} \mathrm{C}$ pendant 20 à $30 \mathrm{~min}$, et précipita par l'acide trichloracétique à une concentration supérieure ou égale à $8 \%$ (Rowland, 1938). Du surnageant obtenu après thermocoagulation de la caséine et des protéines majeures du lactosérum, le traitement par le sulfate d'ammonium à demi-saturation permit à Aschaffenburg (1946) d'isoler une fraction protéique possédant des propriétés tensio-actives : la "Sigma Protéose ». Par le même procédé, en partant de lactosérum de lait emprésuré, Weinstein et al. (1951), isolèrent la "Protéine mineure ». Une autre fraction protéique, également stable thermiquement, fut extraite des membranes de globules gras (Hérald et Brunner, 1957).

L'électrophorèse des protéose-peptones, dans le tube en U de Tiselius permit de distinguer trois fractions protéiques principales (les composants PP3, PP5 et PP8) ainsi nommés dans l'ordre croissant de leur mobilité électrophorétique (Larson et Rolleri, 1955); PP8 étant composé d'une fraction lente (PP8 S) et d'une rapide (PP8 F) (Kolar et Brunner, 1970). Des travaux récents montrèrent que PP5 et PP8 sont des fragments N-terminaux de la caséine $\beta$, complémentaires des caséines $\gamma$ (Andrews, 1978); PP8 F fut obtenu 
par protéolyse in-vitro de cette caséine par la plasmine (Eigel et Keenan, 1979).

L'origine de PP3 est encore inconnue. On sait cependant qu'il présente des réactions immunitaires croisées avec des protéines des membranes de globules gras (Kanno et Yamauchi, 1979). Jusqu'à présent, la séparation des trois composants protéiques consistait simplement en relargages par le sulfate d'ammonium ( $\mathrm{Ng}$ et al., 1970, Kolar et Brunner, 1970) ou par le chlorure de sodium (Jenness, 1959) suivis parfois d'une filtration sur gel (Andrews, 1978).

L'objet du présent travail est, tout d'abord, la comparaison des protéose-peptones obtenues par différents procédés, puis l'élaboration d'une méthode d'isolement de chaque composant protéique, principalement PP3, ainsi que la détermination de quelques caractéristiques moléculaires de ce dernier.

\section{MATERIEL ET METHODES}

\section{Préparation des fractions protéiques}

Elles étaient obtenues à partir soit de lait cru écrémé, soit de

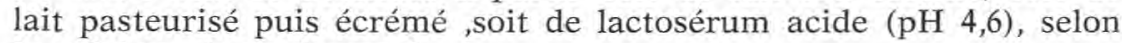
le schéma de la figure 1.

\section{Electrophorèses}

- L'électrophorèse en tube de gel de polyacrylamide était réalisée dans des gels à $9,4 \%$ d'acrylamide $(T=9,4 ; C=4,7$ ) (Hillier, 1976), pour un dépôt de $25 \mu \mathrm{g}$ de produit lyophilisé par tube, avec un appareil Canalco 1200. Après migration, les gels étaient plongés dans l'acide Trichloracétique (TCA) $12 \%$ puis colorés par le bleu de Coomassie R 250 à $0,1 \%$ dans un mélange méthanol : eau; TCA $(1: 1 ; 2 \%)$ et enfin, décolorés dans le mélange méthanol : eau : acide acétique $(3: 6,25: 0,75)$.

- L'électrophorèse sur plaque de gel de polyacrylamide était réalisée au moyen d'un appareil Multiphor 2117 L.K.B. dans un gel à $10,27 \%$ d'acrylamide $(\mathrm{T}=10,27 ; \mathrm{C}=1,36)$ en tampon imidazole $5.10^{-2} \mathrm{M}$, pH 7 contenant $0,1 \%$ de sodium dodecyle sulfate (SDS) selon le procédé préconisé par Fehrnström et Moberg (1977). Les protéines utilisées pour l'étalonnage étaient : $\alpha$-lactalbumine, $\beta$-lactoglobuline, ovalbumine et sérumalbumine.

- La focalisation isoélectrique était réalisée avec un gradient de $\mathrm{pH}(3,5-10)$ dans une plaque de gel à $5,15 \%$ d'acrylamide ( $\mathrm{T}=5,15 ; \mathrm{C}=3,75$ ) en urée $7 \mathrm{M}$ selon Trieu-Cuot et Gripon (1981). Après $3 \mathrm{~h}$ dont $1 \mathrm{~h}$ de préfocalisation, une bande de $1 \mathrm{~cm}$ de largeur coupée dans toute la longueur du gel était divisée en tranches de $0,2 \mathrm{~cm}$, ensuite broyées dans $4 \mathrm{ml}$ d'eau distillée et le $\mathrm{pH}$ de chaque 


\section{MATIERES PREMIERES}

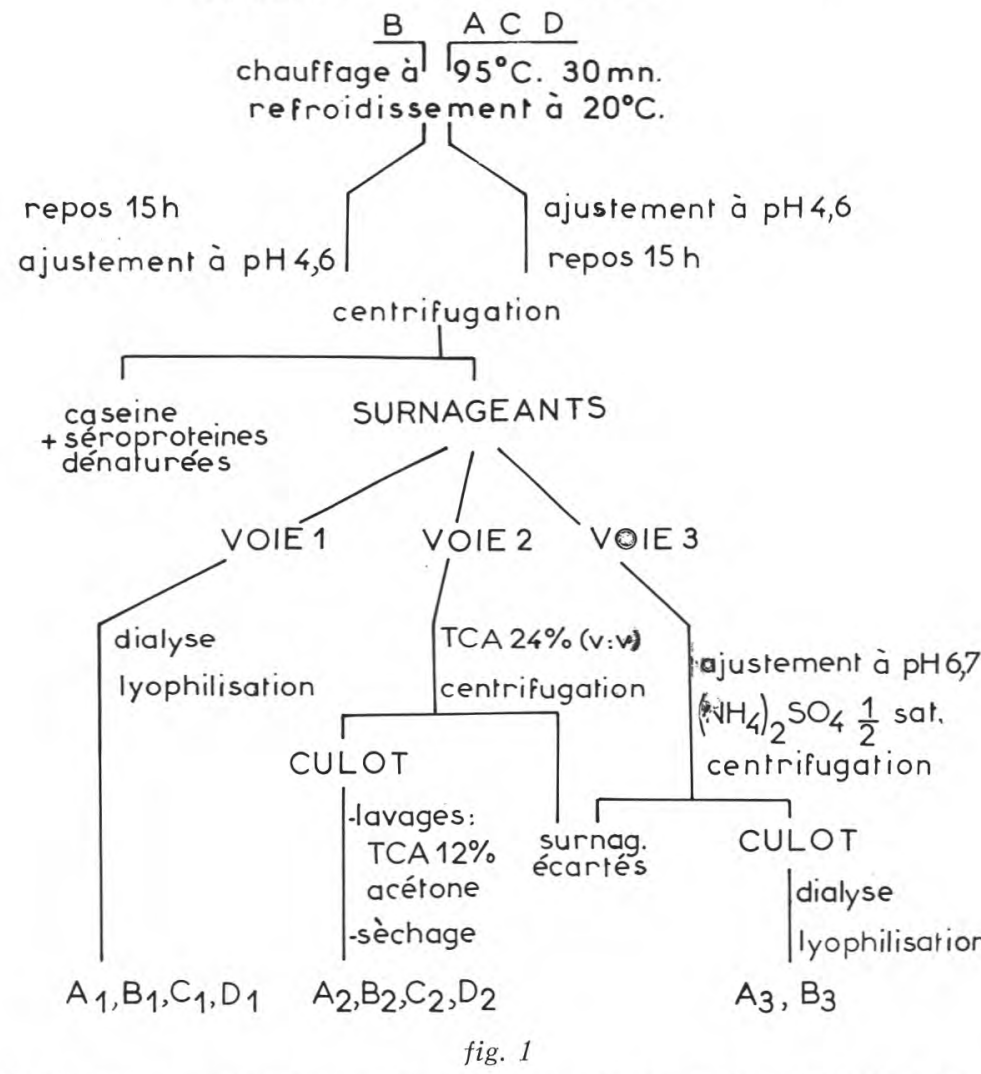

Schéma d'extraction des protéose-peptones à partir de lait écrémé (A et $\mathrm{B}$ ), de lait pasteurisé écrémé (C) et de lactosérum à $\mathrm{pH} 4,6$ (D.). Voie 1 (ROWLAND, 1938), voie 2 (ANDREWS, 1978), voie 3 (ASCHAFFENBURG, 1946).

Extraction diagram of the proteose-peptones from raw skimmilk $(A$ and $B$ ), pasteurized skimmilk $(C)$ and acid whey at $p H 4.6(D)$. Voie 1 (RowLAND, 1938), voie 2 (ANDREWS, 1978), voie 3 (AsCHAFFENBURG, 1946).

substrat mesuré. Le gel était ensuite coloré selon la méthode de Blakesley et Boezi (1977).

Les mesures densitométriques étaient effectuées au moyen d'un densitomètre intégrateur CDS 200 Beckman.

\section{Fractionnements chromatographiques}

Chromatographie d'interactions hydrophobes

Les protéose-peptones lyophilisées (100 ou $200 \mathrm{mg}$ ) en solution dans un tampon phosphate de sodium $10^{-2} \mathrm{M}$, $\mathrm{pH} 6,8$, sulfate d'ammo- 
nium $(0,7$ ou $1 \mathrm{M})$, azide de sodium $0,02 \%$ étaient déposées au sommet d'une colonne $(1,6 \times 15 \mathrm{~cm})$ de phenyl-sepharose $\mathrm{CL} 4 \mathrm{~B}$ équilibrée avec le même tampon. Les protéines étaient éluées par un gradient décroissant de sulfate d'ammonium réalisé au moyen d'un appareil Ultrograd 11300 L.K.B. avec un débit de $20 \mathrm{ml} / \mathrm{h}$. Les fractions obtenues étaient dialysées puis concentrées.

\section{Filtration sur gel}

La fraction (j) obtenue précédemment, était dialysée contre un tampon Tris-HCl $0,1 \mathrm{M}, \mathrm{pH} 8 \mathrm{NaCl} 0,5 \mathrm{M}$, azide de sodium $0,02 \%$ puis déposée ( 13 $\mathrm{mg}$ de protéine) au sommet d'une colonne $(1,6 \times 46 \mathrm{~cm})$ de Séphacryl S 200 équilibrée avec le même tampon. L'élution était menée avec un débit de $32 \mathrm{ml} / \mathrm{h}$.

\section{Analyses}

L'analyse quantitative des acides aminés était réalisée après une hydrolyse des échantillons ( 6 et $24 \mathrm{~h}, \mathrm{HCl} 6 \mathrm{~N}, 110^{\circ} \mathrm{C}$, sous vide) à l'aide d'un Auto-analyseur Technicon NC II P.

L'acide aminé N-terminal était identifié par dansylation selon Wood et Wang (1967).

Le phosphore était dosé par la méthode de Bamman et al., (1948).

\section{RESULTATS}

\section{Extraction des protéose-peptones}

Les fractions protéiques ont été examinées par électrophorèse en tube de gel de polyacrylamide (fig. 2). Quelle que soit la méthode employée (fig. 1), on observe un même profil : 3 bandes majeures et de nombreuses bandes mineures; la bande la plus lente correspond à PP3 et la bande très large de mobilité moyenne à PP5. PP8 F migre au niveau du front alors que PP8 S se présente sous forme d'un doublet migrant juste derrière.

On remarque dans les produits $A_{1}, A_{2}$ et $A_{3}$ une bande migrant un peu en avant de PP3, dont on ne connaît pas la nature. Elle est peu visible dans les produits B (même lait, mais temps de séjour différent à $\mathrm{pH} 4,6$ ), C (lait pasteurisé) et $\mathrm{D}$ (lactosérum).

Pour le lactosérum (D), le nombre de bandes est un peu moins grand que pour les laits (A, B, C) mais on retrouve les bandes principales.

Le traitement des protéose-peptones dans le gel de polyacrylamide par le réactif de Schiff ne colore que la bande correspondant à PP3 (expérience non montrée ici). 

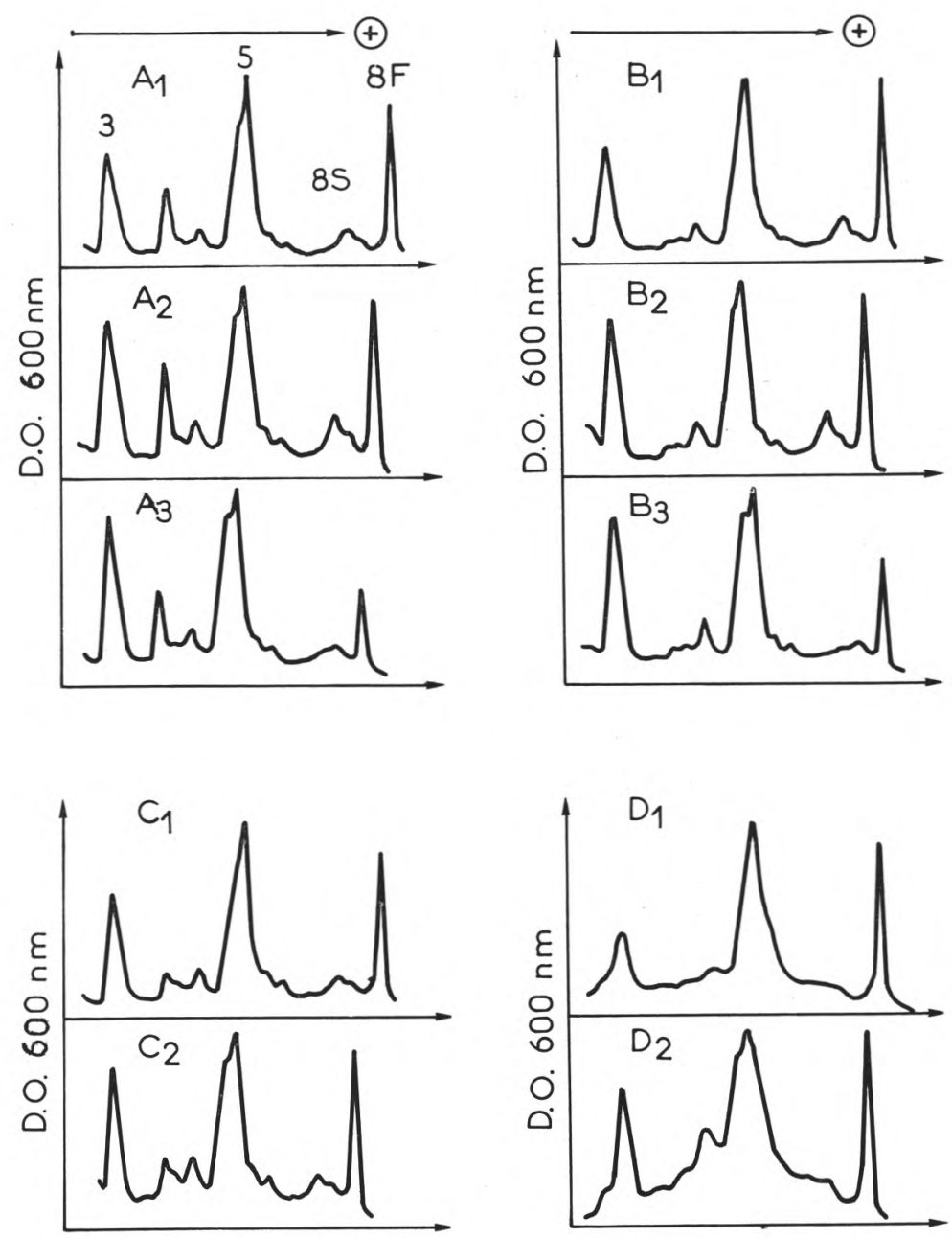

fig. 2

Profils densitométriques des électrophorèses, en tubes de gel de polyacrylamide 9,4 \%, pH 8,9, des protéose-peptones obtenues selon le schéma de la figure 1 .

Densitometric diagrams of polyacrylamide disc electrophoresis at 9,4\% and $p H 8.9$ of the proteose-peptones obtained with the diagram of fig. 1. 


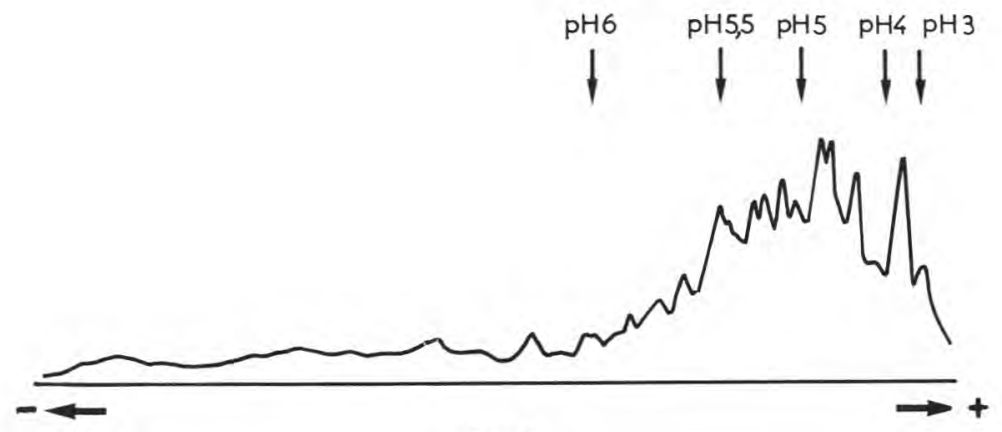

fig. 3

Profil densitométrique de la focalisation isoélectrique d'une protéosepeptone entre $\mathrm{pH} 3,5$ et 10 .

Densitometric diagram of electrofocusing of a proteose-peptones between pH 3,5-10.

La focalisation isoélectrique entre $\mathrm{pH}$ 3,5 et 10 (fig. 3) montre un grand nombre de bandes protéiques localisées principalement entre pH 3 et 6. Par contre, l'électrophorèse sur plaque de gel de polyacrylamide en présence de sodium dodecyle sulfate ne montre que 3 bandes dont les poids moléculaires respectifs seraient : 15800 , 21500,31600 .

\section{Fractionnement des protéose-peptones}

La chromatographie sur colonne de phényl-sepharose (fig. 4.1.) permet de fractionner les protéose-peptones en leurs différents composants protéiques. Le lavage par le tampon de départ élue des substances non absorbées sur le support; la fraction (a) contenant essentiellement des protéines de grande mobilité électrophorétique. Au début du gradient sont éluées les fractions (b), (c) et (d) contenant peu de protéines, bien que (c) ait une assez forte absorption à $280 \mathrm{~nm}$. La fraction (e) éluée au milieu du gradient contient PP5. Les fractions $(f+g)$ à la fin $d u$ gradient contiennent PP3 (fig. 5.1.). La modification du gradient (fig. 4.2.) $(0,7 \mathrm{M}-0$ au lieu de 1 M-0) comportant 2 pentes successives $(-9,4$ puis -7 mmoles $\left(\mathrm{NH}_{4}\right)_{2} \mathrm{SO}_{4} / \mathrm{ml}$ ) permet de bien séparer PP5 et PP3 (fig. 5.2.). Cette dernière chromatographie a pu être répétée au moins quatre fois avec la même colonne, en déposant $200 \mathrm{mg}$ de protéose-peptones lyophilisées, sans altérer la reproductibilité de l'élution (fig. 4.3.).

La fraction (j) obtenue précédemment a été chromatographiée sur une colonne de gel Sephacryl S 200. Le diagramme d'élution 

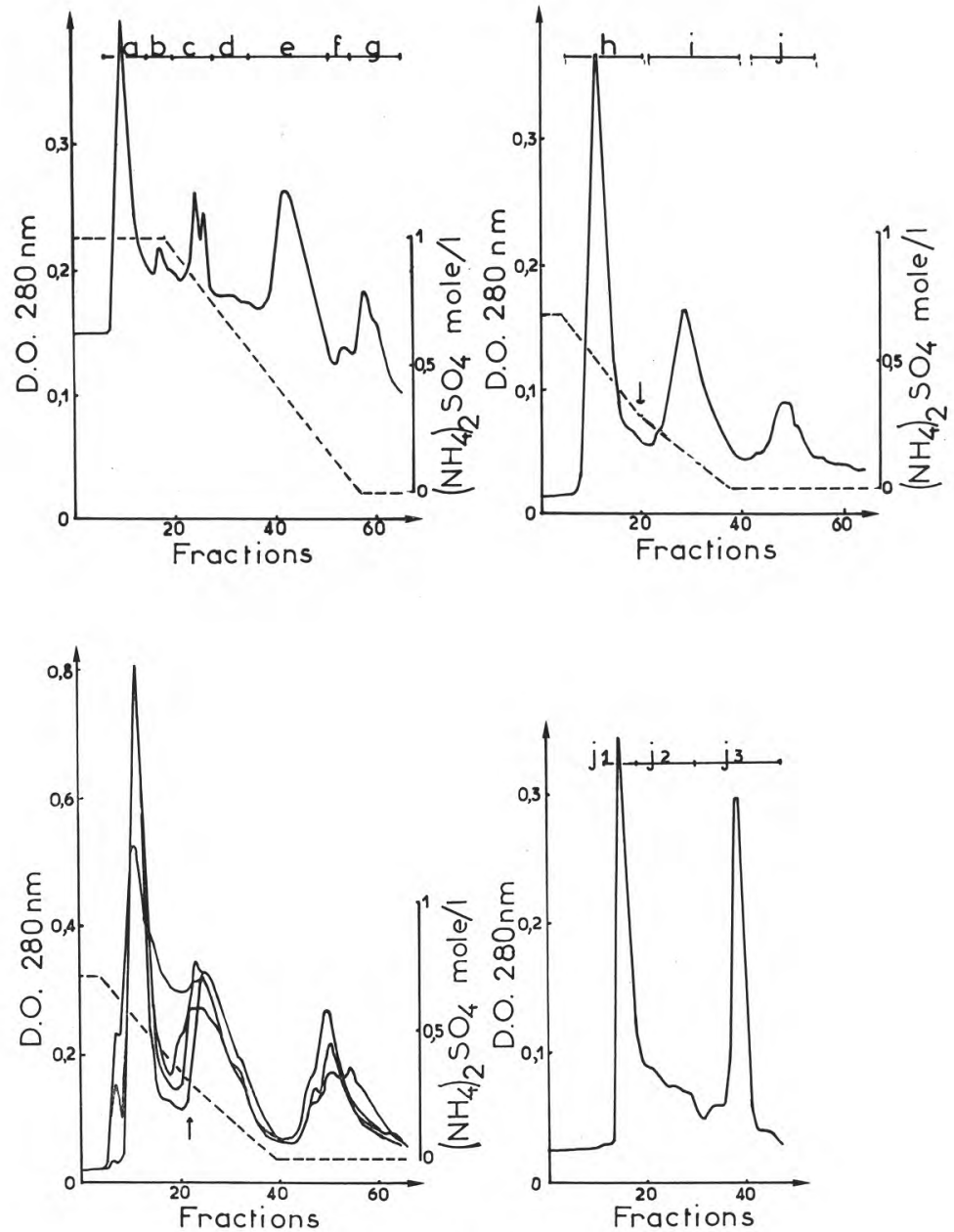

fig. 4

Chromatographie des protéose-peptones sur colonne de phényl-sèpharose CL 4B (fig. 4.1, 4.2 et 4.3) et filtration sur Sephacryl S 200 du composant-3 (fig. 4.4).

Chromatography on phenyl-sepharose $C L 4 B$ of proteose-peptones (4.1, 4.2 and 4.3) and gel filtration of component-3 on Sephacryl S 200 (4.4).

(fig. 4.4.) montre un premier pic ,totalement exclu du gel, qui contient PP3; on trouve dans la fraction suivante $(\mathrm{j} 2)$ les protéines contaminantes (fig. 5.3.). La fraction (j3) ne donne pas de bande de protéine. 

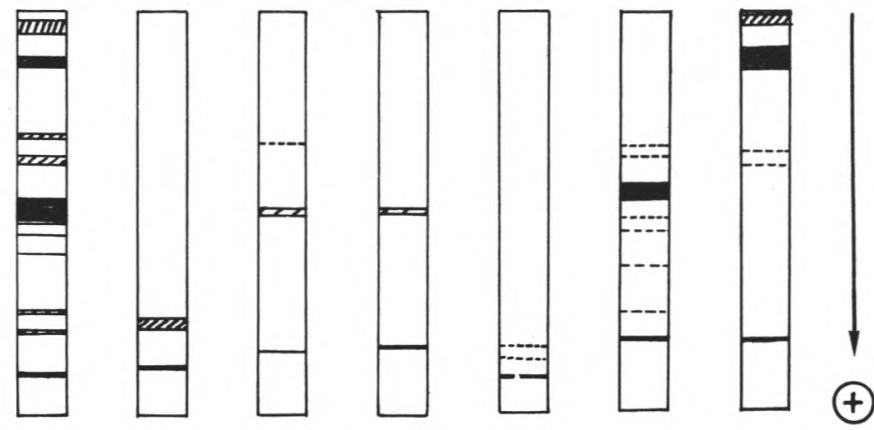

$\mathrm{T}$

a

b

c

d

e

$f+g$
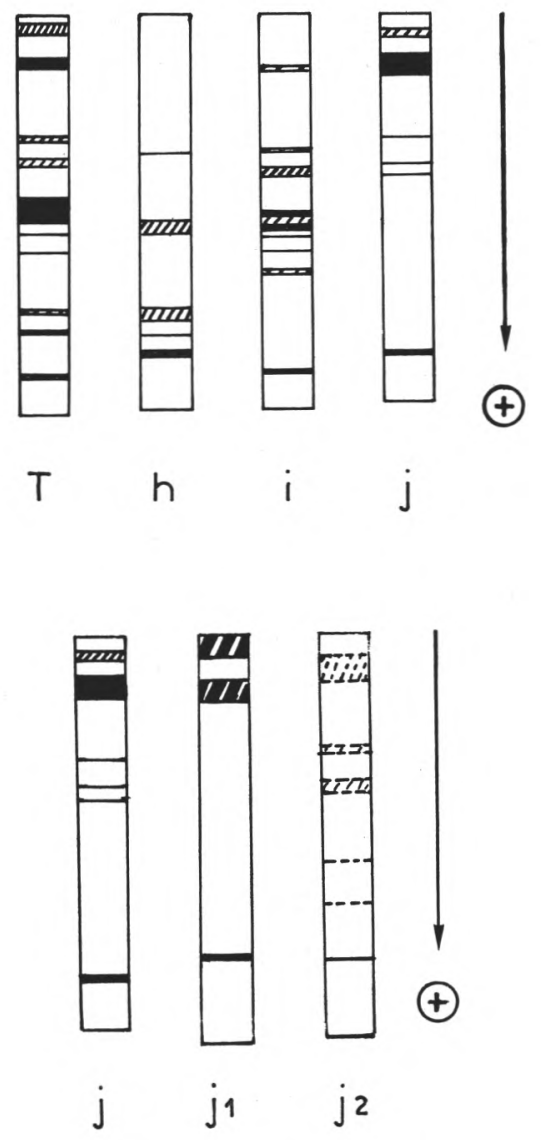

fig. 5

Diagrammes représentant l'électrophorèse en tubes de gel de polyacrylamide des fractions protéiques éluées sur les colonnes de phénylsépharose CL 4B (4.1 et 4.2) et la colonne de Sephacryl S 200.

Diagrammatic representation of polyacrylamide disc-electroporesis patterns obtained from protein fractions eluted from phenyl-sepharose CL 4B column (4.1 and 4.2) and from Sephacryl S 200 column (4.3). 


\section{Caractéristiques moléculaires de PP3}

La composition en acides aminés est montrée dans le tableau 1. On peut voir que PP3 contient plus de sérine et de glycine que les protéose-peptones en général, par contre, il contient moins de proline et de valine. Comme les protéose-peptones, ce composant contient peu d'acides aminés aromatiques (la cystéine et le tryptophane n'ont pas été déterminés).

L'électrophorèse sur plaque de gel de polyacrylamide en présence de sodium dodecyle sulfate indique un poids moléculaire de $21400 \pm$ 1000 . L'identification de l'acide $\mathrm{N}$-terminal par dansylation donne la sérine. Le taux de phosphore dosé par rapport à la partie protéique de la molécule est $1,1 \%$.

\section{TABLEAU 1 - TABLE 1}

Composition en acides aminés du composant-3 (PP3) et de différentes protéose-peptones $\left(A_{1}, A_{2}, A_{3}\right)$ (résultats en moles \%)

Amino-acid composition of component-3 (PP3) and various proteose-peptones

$\left(A_{1}, A_{2}, A_{3}\right)$ (results in moles \%)

\begin{tabular}{|c|c|c|c|c|}
\hline & PP3 & $A_{1}$ & $\mathrm{~A}_{2}$ & $\mathrm{~A}_{3}$ \\
\hline ASP & 9,3 & 9,2 & 8,6 & 8,4 \\
\hline THR & 7,7 & 6,1 & 5,8 & 6,1 \\
\hline SER & 12,7 & 8,5 & 8,1 & 8,0 \\
\hline GLU & 15,3 & 19,7 & 18,8 & 18,4 \\
\hline PRO & 4,9 & 10,5 & 11,5 & 12,1 \\
\hline GLY & 7,7 & 3,0 & 2,8 & 3,1 \\
\hline ALA & 5,2 & 3,9 & 3,6 & 3,9 \\
\hline VAL & 3,5 & 6,8 & 6,5 & 6,7 \\
\hline MET & 1,7 & 1,1 & 1,6 & 1,7 \\
\hline ILE & 5,2 & 6,5 & 6,5 & 6,1 \\
\hline LEU & 9,5 & 9,2 & 9,2 & 8,3 \\
\hline TYR & 1,0 & 1,1 & 1,4 & 1,2 \\
\hline PHE & 2.2 & 3,1 & 3,2 & 3,7 \\
\hline LYS & 7,7 & 7,1 & 7,2 & 7,2 \\
\hline HIS & 3,5 & 2,6 & 2,6 & 2,6 \\
\hline ARG & 3,3 & 2,4 & 2,6 & 2,5 \\
\hline
\end{tabular}

\section{DISCUSSION}

Les fractions protéiques isolées du surnageant après thermocoagulation des protéines majeures du lait ou du lactosérum ne 
présentent apparemment pas de différences dans leurs images électrophorétiques. Seul, PP3 présente la coloration caractéristique des glycoprotéines; PP5 et PP8 ne seraient donc pas des glycoprotéines, ce qui rejoint les conclusions de Andrews (1978), et ceci malgré les résultats de Kolar et Brunner (1970).

L'électrophorèse en présence de sodium dodecyle sulfate montre seulement 3 bandes; la plus rapide a un poids moléculaire moyen de 15800 , proche de celui trouvé par Andrews (1978) pour PP5. PP3 correspond à la bande de mobilité moyenne, nous n'avons pas identifié la bande lente (PM 31600 ). Les conditions de l'électrophorèse (acrylamide $10 \%$ ) ne permettaient pas de détecter PP8 F et $\mathrm{S}$ de poids moléculaires respectifs $3600 \pm 300$ (Andrews, 1978) et 8800 (Eigel et Keenan, 1979).

Au cours de la chromatographie d'interactions hydrophobes les substances non adsorbées sur le gel ont de fortes mobilités électrophorétiques (fig. 5.1.a.) et pourraient correspondre à PP8 S et en particulier PP8 F qui selon Andrews (1978) a la même séquence que le fragment $\mathrm{N}$-terminal $1-28$ de la caséine $\beta$ et ne contient pas de résidu d'acide aminé aromatique; il ne serait pas retenu sur le gel par l'intermédiaire d'interaction de type $\pi-\pi$. Cette chromatographie permet aussi de voir que PP3 est le plus hydrophobe des constituants des protéose-peptones. Cette méthode peut donc aisément être employée pour isoler l'un ou l'autre des composants à partir du surnageant de thermocoagulation sans recourir à une série de précipitations par les sels, et permet d'obtenir PP3 dans un état de pureté élevée.

PP3, dont le poids moléculaire est 22000 (évalué par électrophorèse en présence de SDS) est élué dans le volume mort de la colonne de gel de Sephacryl S 200 qui a un domaine d'exclusion approximatif de 250000 . Ce résultat montre que cette molécule peut se comporter comme un polymère dans les conditions de cette expérience (tampon Tris, $\mathrm{pH}$ 8). Ceci va dans le même sens que les résultats de $\mathrm{Ng}$ et al. (1970), qui, par ultracentrifugation, ont trouvé des poids moléculaires de 163000 en tampon véronal pH 8,6 et de 40000 dans le même tampon en présence de guanidine $5 \mathrm{M}$.

La composition en acides aminés des protéose-peptones est caractérisée par le taux important de proline et la faible quantité de résidus aromatiques. $\mathrm{PP} 3$, lui, se distingue par une teneur élevée en sérine et en glycine et par une faible teneur en proline. Dans les protéose-peptones globales ,les résidus proline sont apportés principalement par PP5 et PP8.

PP3 est une phosphoglycoprotéine, la partie glucidique de la molécule est actuellement à l'étude ainsi que les séquences $\mathrm{N}$ et C-terminales. 


\section{Bibliographie}

ANDREWS (A. T.) (1978). - The composition, structure and origin of proteosepeptone component 5 of bovine milk. Eur. J. Biochem., 90, 59-65.

ANDREWS (A. T.) (1978). - The composition, structure and origin of proteosepeptone component $8 \mathrm{~F}$ of bovine milk. Eur. J. Biochem., 90, 67-71.

Aschaffenburg (R.) (1946). - Surface activity and proteins of milk. J. Dairy Res., 14, 316-329.

Bamman (E.), Novotny (E.) und Rohr (L.) (1948). - Zur colorimetrischer Bestimmung der Phosphosaüre. Chem. Ber., 81, 438-449.

Blakesley (R.W.) and Boezi (J. A.) (1977). - A new staining technique for proteins in polyacrylamide gels using coomassie brillant blue G 250. Anal. Biochem., 82, 580-582.

EIGeL (W. W.) and KEENAN (T. W.) (1979). - Identification of proteose-peptone component 8 slow as a plasmin derived fragment of bovine $\beta$-casein. Int. J. Biochem., 10, 529-535.

FehrnströM (H.) and MoBerg (U.) (1977). - Analytical proteins separation. Application note 306 L.K.B. Produkter AB, Bromma, Suede.

Herald (C. T.) and BRunNer (J. R.) (1957). - The fat-globule membrane of normal cow's milk. I. The isolation and characteristics of two membraneprotein fractions, J. Dairy Sci., 40, 948-956.

Hillier (R. M.) (1976). - The quantitative measurement of whey proteins using polyacrylamide-gel electrophoresis. J. Dairy Res., 43, 259-265.

Jenness (R.) (1959). - Characterization of milk serum component 5. J. Dairy Sci., 42, 895.

Kanno (C.) and Yamauchi (K.) (1979). - Relationship of soluble glycoprotein of milk fat globule membrane to component-3, -5 and -8 fractions of proteose-peptone. Agric. Biol. Chem., 43, 2105-2113.

Kolar (C. W.) and BRUnner (J.R.) (1970). - Proteose-peptone fraction of bovine milk: Lacteal serum components-5 and -8 casein associated glycoproteins. J. Dairy Sci., 53, 997-1008.

LARSON (B. L.) and Rolleri (G. D.) (1955). - Heat denaturation of the specific serum proteins in milk. J. Dairy Sci., 38, 351-360.

NG (W. C.), Brunner (J. R.) and Rhee (K. C.) (1970). - Proteose-peptone fraction of bovine milk: Lacteum serum component-3. A whey glycoprotein. J. Dairy Sci., 53, 987-996.

Rowland (S. J.) (1938), - The precipitation of the proteins in milk. I. Casein. II. Total proteins. III. Globulin. IV. Albumin and proteose-peptone, J. Dairy Res., 9, 30-41.

Trieu-Cuot (P.) and Gripon (J. C.) (1981). - Electrofocusing and two dimensional electrophoresis of bovine caseins. J. Dairy Res., 48, 303-310.

Weinstein (B. R.), Lillevik (H. A.), Duncan (C. W.) and Trout (G. M.) 1951). The solar activated flavor of homogenized milk. V. The electrophoretic analysis of a contributing minor protein fraction. J. Dairy Sci., 34, 784-789.

Woods (K. R.) and WANG (K. T.) (1967). - Separation of dansyl-amino acids by polyamide layer chromatography. Biochim. Biophys. Acta, 133, 369-370. 